\title{
Multilinear Singular Integrals and their Commutators with Nonsmooth Kernels on Weighted Morrey Spaces
}

\author{
Songbai Wang and Yinsheng Jiang \\ College of Mathematics and System Sciences, Xinjiang University, Urumqi 830046, China \\ Correspondence should be addressed to Yinsheng Jiang; ysjiang@xju.edu.cn \\ Received 21 October 2013; Accepted 4 December 2013 \\ Academic Editor: Alberto Fiorenza
}

Copyright (c) 2013 S. Wang and Y. Jiang. This is an open access article distributed under the Creative Commons Attribution License, which permits unrestricted use, distribution, and reproduction in any medium, provided the original work is properly cited.

Some multilinear maximal functions and the generalized Calderón-Zygmund operators and their commutators with nonsmooth kernels are studied. The purpose of this paper is to establish that these operators are bounded on certain product Morrey spaces $L^{p, k}\left(\mathbb{R}^{n}\right)$. Based on the boundedness of these operators from $L^{p_{1}}\left(\omega_{i}\right) \times \cdots \times L^{p_{m}}\left(\omega_{m}\right)$ to $L^{p}\left(\prod_{j=1}^{m} \omega_{j}^{p / p_{j}}\right)$, we obtained that they are also bounded from $L^{p_{1}, k}\left(\omega_{i}\right) \times \cdots \times L^{p_{m}, k}\left(\omega_{m}\right)$ to $L^{p, k}\left(\prod_{j=1}^{m} \omega_{j}^{p / p_{j}}\right)$ with $0<k<1,1<p_{j}<\infty, 1 / p=1 / p_{1}, \ldots, p_{m}$ and $\omega_{j} \in A_{p_{j}}$, $j=1, \ldots, m$.

\section{Introduction}

Let $\mathcal{S}\left(\mathbb{R}^{n}\right)$ and $\mathcal{S}^{\prime}\left(\mathbb{R}^{n}\right)$ be the Schwartz spaces of all rapidly decreasing functions and tempered distributions, respectively. Let $T$ be a multilinear operator initially defined on the $m$-fold product of Schwartz spaces and taking values into the space of tempered distributions,

$$
T: \mathcal{S}\left(\mathbb{R}^{n}\right) \times \cdots \mathcal{S}\left(\mathbb{R}^{n}\right) \longrightarrow \mathcal{S}^{\prime}\left(\mathbb{R}^{n}\right) .
$$

Following [1], the $m$-multilinear Calderón-Zygmund operator $T$ satisfies the following conditions:

(S1) there exist $q_{i}<\infty(i=1, \ldots, m)$, it extends to such that a bounded multilinear operator from $L^{q_{1}} \times \cdots \times$ $L^{q_{m}}$ to $L^{q}$, where

$$
\frac{1}{q}=\frac{1}{q_{1}}+\cdots+\frac{1}{q_{m}}
$$

(S2) there exists a function $K$, defined off the diagonal $x=$ $y_{1}=\cdots=y_{m}$ in $\left(\mathbb{R}^{n}\right)^{m+1}$, satisfying

$$
\begin{aligned}
T(\vec{f})(x)= & T\left(f_{1}, \ldots, f_{m}\right)(x) \\
= & \int_{\left(\mathbb{R}^{m}\right)^{n}} K\left(x, y_{1}, \ldots, y_{m}\right) f_{1}\left(y_{1}\right) \ldots \\
& f_{m}\left(y_{m}\right) d y_{1} \ldots d y_{m},
\end{aligned}
$$

for all $x \notin \cap_{j=1}^{m} \operatorname{supp} f_{j}$ and $f_{1}, \ldots, f_{m} \in \mathcal{S}\left(\mathbb{R}^{n}\right)$, where

$$
\begin{aligned}
& \left|K\left(y_{0}, y_{1}, \ldots, y_{m}\right)\right| \leq \frac{A}{\left(\sum_{l, k=0}^{m}\left|y_{l}-y_{k}\right|\right)^{m n}} \\
& \left|K\left(y_{0}, \ldots, y_{j}, \ldots, y_{m}\right)-K\left(y_{0}, \ldots, y_{j}, \ldots, y_{m}\right)\right| \\
& \quad \leq \frac{A\left|y_{j}-y_{j}^{\prime}\right|^{\epsilon}}{\left(\sum_{l, k=0}^{m}\left|y_{l}-y_{k}\right|\right)^{m n+\epsilon}},
\end{aligned}
$$

for some $\epsilon>0$ and all $0 \leq j \leq m$, whenever $\left|y_{j}-y_{j}\right| \leq$ $(1 / 2) \max _{0 \leq k \leq m}\left|y_{j}-y_{k}\right|$.

We also take some notation following [2]. Given a locally integrable vector function $\mathbf{b}=\left(b_{1}, \ldots, b_{m}\right) \in(\mathrm{BMO})^{m}$. The commutator of $\mathbf{b}$ and the $m$-linear Calderón-Zygmund operator $T$, denoted here by $T_{\Sigma \mathbf{b}}$, was introduced by Pérez and Torres in [3] and is defined via

$$
T_{\Sigma \vec{b}}(\vec{f})=\sum_{j=1}^{m} T_{b_{j}}^{j}(\vec{f})
$$

where

$$
T_{b_{j}}^{j}(\vec{f})=b_{j} T(\vec{f})-T\left(f_{1}, \ldots, b_{j} f_{j}, \ldots, f_{m}\right) .
$$


And the iterated commutators $T_{\Pi \mathbf{b}}$ are defined by

$$
T_{\Pi \vec{b}}(\vec{f})=\left[b_{1}, \ldots,\left[b_{m-1},\left[b_{m}, T\right]_{m}\right]_{m-1} \ldots\right]_{1}(\vec{f}) .
$$

To clarify the notations, if $T$ is associated in the usual way with a Calderón-Zygmund kernel $K$, then at a formal level

$$
\begin{aligned}
& T_{\Sigma \vec{b}}(\vec{f})(x)=\int_{\left(\mathbb{R}^{n}\right)^{m}} \sum_{j=1}^{m}\left(b_{j}(x)-b_{j}\left(y_{j}\right)\right) \\
& \times K\left(x, y_{1}, \ldots, y_{m}\right) f_{1}\left(y_{1}\right) \ldots \\
& f_{m}\left(y_{m}\right) d y_{1} \cdots d y_{m}, \\
& T_{\Pi \vec{b}}(\vec{f})(x)=\int_{\left(\mathbb{R}^{n}\right)^{m}} \prod_{j=1}^{m}\left(b_{j}(x)-b_{j}\left(y_{j}\right)\right) \\
& \times K\left(x, y_{1}, \ldots, y_{m}\right) f_{1}\left(y_{1}\right) \cdots \\
& \quad f_{m}\left(y_{m}\right) d y_{1} \cdots d y_{m} .
\end{aligned}
$$

In this paper, we will consider $T$ to be associated with the kernel satisfying a weaker regularity conditions introduced by $[4,5]$. A special example is the $m$ th Calderón commutator.

Let $\left\{A_{t}\right\}_{t>0}$ be a class of integral operators, which play the role of the approximation to the identity. We always assume that the operators $A_{t}$ are given by kernels $a_{t}(x, y)$ in the sense that

$$
A_{t} f(x)=\int_{\mathbb{R}^{n}} a_{t}(x, y) f(y) d y,
$$

for all $f \in \cup_{p \in[1, \infty]} L^{p}$ and $x \in \mathbb{R}^{n}$, and the kernels $a_{t}(x, y)$ satisfy the following conditions:

$$
\left|a_{t}(x, y)\right| \leq h_{t}(x, y):=t^{-n / s} h\left(\frac{|x-y|}{t^{1 / s}}\right),
$$

where $s$ is a positive fixed constant and $h$ is a positive, bounded, decreasing function satisfying that for some $\eta>0$

$$
\lim _{r \rightarrow \infty} r^{n+\eta} h\left(r^{s}\right)=0 \text {. }
$$

Recall that the $j$ th transpose $T^{*, j}$ of the $m$-linear operator $T$ is defined via

$$
\begin{aligned}
& \left\langle T^{*, j}\left(f_{1}, \ldots, f_{m}\right), g\right\rangle \\
& \quad=\left\langle T^{*, j}\left(f_{1}, \ldots, f_{j-1}, g, f_{j+1}, \ldots, f_{m}\right), f_{j}\right\rangle,
\end{aligned}
$$

for all $f_{1}, \ldots, f_{m}, g$ in $\mathcal{S}\left(\mathbb{R}^{n}\right)$. It is seen that the kernel $K^{*, j}$ of $T^{*, j}$ is related to the kernel $K$ of $T$ via the identity

$$
\begin{aligned}
& K^{*, j}\left(x, y_{1}, \ldots, y_{j-1}, y_{j}, y_{j+1}, \ldots, f_{m}\right) \\
& \quad=K\left(y_{j}, y_{1}, \ldots, y_{j-1}, x, y_{j+1}, \ldots, f_{m}\right) .
\end{aligned}
$$

If an $m$-linear operator $T$ maps a product of Banach spaces $X_{1} \times \cdots \times X_{m}$ into another Banach space $X$, then the transpose $T^{*, j}$ maps $X_{1} \times \cdots \times X_{j-1} \times X \times X_{j+1} \times \cdots \times X_{m}$ to $X_{j}$. Moreover, the norms of $T$ and $T^{*, j}$ are equal. To maintain uniform notation, we may occasionally denote $T$ by $T^{*, 0}$ and $K$ by $K^{*, 0}$.
Assumption 1. Assume that for each $i=1, \ldots, m$ there exist operators $\left\{A_{t}^{(i)}\right\}_{t>0}$ with kernels $a_{t}^{(i)}(x, y)$ that satisfy conditions (11) and (12) with constants $s$ and $\eta$ and that, for every $j=0,1,2, \ldots, m$, there exist kernel $K^{*, j,(i)}\left(x, y_{1}, \ldots, y_{m}\right)$ such that

$$
\begin{gathered}
\left\langle T^{*, j}\left(f_{1}, \ldots, A_{t}^{(i)} f_{i}, \ldots, f_{m}\right), g\right\rangle \\
=\int_{\mathbb{R}^{n}} \int_{\left(\mathbb{R}^{n}\right)^{m}} K_{t}^{*, j,(i)}\left(x, y_{1}, \ldots, f_{m}\right) f_{1}\left(y_{1}\right) \ldots \\
f_{m}\left(y_{m}\right) g(x) d y_{1} \cdots d y_{m} d x
\end{gathered}
$$

for all $f_{1}, \ldots, f_{m}, g$ in $\mathcal{S}\left(\mathbb{R}^{n}\right)$ with $\cap_{k=1}^{m}$ supp $f_{k} \cap$ supp $g=$ $\emptyset$. Also assume that there exist a function $\phi \in \mathscr{C}(\mathbb{R})$ with supp $\phi \subset[-1,1]$ and constants $\epsilon>0$ and $A$ so that for every $j=0,1,2, \ldots, m$ and every $i=1,2, \ldots, m$, we have

$$
\begin{gathered}
\left|K^{*, j}\left(x, y_{1}, \ldots, y_{m}\right)-K_{t}^{*, j,(i)}\left(x, y_{1}, \ldots, y_{m}\right)\right| \\
\leq \frac{A}{\left(\sum_{k=1}^{m}\left|x-y_{k}\right|\right)^{m n}} \sum_{k=1, k \neq i}^{m} \phi\left(\frac{\left|y_{i}-y_{k}\right|}{t^{1 / s}}\right) \\
\quad+\frac{A t^{\epsilon / t}}{\left(\sum_{k=1}^{m}\left|x-y_{k}\right|\right)^{m n+\epsilon}}
\end{gathered}
$$

whenever $t^{1 / s} \leq\left|x-y_{i}\right| / 2$.

If $T$ satisfies Assumption 1 we will say that $T$ is an $m$ linear operator with generalized Calderón-Zygmund kernel $K$. The collection of function $K$ satisfying (15) and (16) with parameters $m, A, s, \eta$, and $\epsilon$ will be denoted by $m$-linear $\operatorname{GCZK}(A, s, \eta, \epsilon)$. We say that $T$ is of class $m-G C Z O(A, s, \eta, \epsilon)$ if $T$ has an associated kernel $K$ in $m-G C Z K(A, s, \eta, \epsilon)$. Throughout this paper, we always assume that the $m$-linear operator $T$ satisfies the following assumption.

Assumption 2. Assume that there exist some $1 \leq q_{1}, \ldots, q_{m}<$ $\infty$ and some $0<q<\infty$ with $1 / q=1 / q_{1}+\cdots+1 / q_{m}$, such that $T$ maps $L^{q_{1}}\left(\mathbb{R}^{n}\right) \times \cdots \times L^{q_{m}}\left(\mathbb{R}^{n}\right)$ to $L^{q, \infty}\left(\mathbb{R}^{n}\right)$.

Theorem 3 (see [4]). Assume that $T$ is a multilinear operator in $m-G C Z O(A, s, \eta, \epsilon)$. Let $1 / m \leq p<\infty, 1 \leq p_{j} \leq \infty$ with $1 / p=1 / p_{1}+\cdots+1 / p_{m}$, all the following statement are valid:

(i) when all $p_{j}>1$, then $T$ can be extended to be a bounded operator from the $m$-fold product $L^{p_{1}}\left(\mathbb{R}^{n}\right) \times$ $\cdots \times L^{p_{m}}\left(\mathbb{R}^{n}\right)$ to $L^{p}\left(\mathbb{R}^{n}\right)$;

(ii) when some $p_{j}=1$, then $T$ can be extended to be a bounded operator from the $m$-fold product $L^{p_{1}}\left(\mathbb{R}^{n}\right) \times$ $\cdots \times L^{p_{m}}\left(\mathbb{R}^{n}\right)$ to $L^{p, \infty}\left(\mathbb{R}^{n}\right)$.

Moreover, there exists a constant $C\left(n, m, p_{j}, q_{j}\right)$ such that

$$
\begin{aligned}
& \|T\|_{L^{1} \times \cdots \times L^{1} \rightarrow L^{1 / m, \infty}} \\
& \quad \leq C\left(n, m, p_{j}, q_{j}\right)\left(A+\|T\|_{L^{q_{1} \times \cdots \times L^{q_{m}}} \rightarrow L^{q, \infty}}\right) .
\end{aligned}
$$


Assumption 4. Assume that there exist operators $\left\{B_{t}\right\}_{t>0}$ with kernels $b_{t}(x, y)$ that satisfy condition (11) and (12) with constants $s$ and $\eta$. Let

$$
K_{t}^{(0)}\left(x, y_{1}, \ldots, y_{m}\right)=\int_{\mathbb{R}^{n}} K\left(z, y_{1}, \ldots, y_{m}\right) b_{t}(x, z) d z
$$

We assume that the kernels $K_{t}^{(0)}\left(x, y_{1}, \ldots, y_{m}\right)$ satisfy the following estimates; there exist a function $\phi \in \mathscr{C}(\mathbb{R})$ with supp $\phi \subset[-1,1]$ and constants $\epsilon>0$ and $A$ such that

$$
\left|K_{t}^{(0)}\left(x, y_{1}, \ldots, y_{m}\right)\right| \leq \frac{A}{\left(\sum_{k=1}^{m}\left|x-y_{k}\right|\right)^{m n}}
$$

whenever $2 t^{1 / s} \leq \min _{1 \leq j \leq m}\left|x-y_{j}\right|$, and

$$
\begin{aligned}
& \left|K\left(x, y_{1}, \ldots, y_{m}\right)-K_{t}^{(0)}\left(x^{\prime}, y_{1}, \ldots, y_{m}\right)\right| \\
& \leq \frac{A}{\left(\sum_{k=1}^{m}\left|x-y_{k}\right|\right)^{m n}} \sum_{k=1}^{m} \phi\left(\frac{\left|y_{i}-y_{k}\right|}{t^{1 / s}}\right) \\
& \quad+\frac{A t^{\epsilon / s}}{\left(\sum_{k=1}^{m}\left|x-y_{k}\right|\right)^{m n+\epsilon}},
\end{aligned}
$$

whenever $2\left|x-x^{\prime}\right| \leq t^{1 / s} \leq \max _{1 \leq j \leq m}\left|x-y_{j}\right| / 2$.

It is known that condition (16) is weaker than, and indeed a consequence of, the Calderón-Zygmund kernel condition (5) from the proof of Proposition 2.1 in [4]. And also it is pointed out that Assumption 4 is weaker than the condition (5) for $K\left(x, y_{1}, \ldots, y_{m}\right)$ in [6].

For $T$ be an $m$-linear Calderón-Zygmund operator, $\vec{\omega} \epsilon$ $A_{\vec{p}}$ and $\nu_{\vec{\omega}}=\prod_{j=1}^{m} \omega_{j}^{p / p_{j}}$ with $1 / p=1 / p_{1}+\cdots+p_{m}$ and $\vec{b} \epsilon$ $B M O^{m}$, Lerner et al. [7] proved that $T$ and $T_{\Sigma \vec{b}}$ bounded from $L^{p_{1}}\left(\omega_{1}\right) \times \cdots \times L^{p_{m}}\left(\omega_{m}\right)$ to $L^{p}\left(\nu_{\vec{\omega}}\right)$ and Pérez et al. [2] extended the result to $T_{\Pi \vec{b}}$ when all $1<p_{j}<\infty$, in the case of the endpoint, that is, some $p_{j}=1$, weak type estimates have been established; for some details refer [2,7]. To obtain the same results for the multilinear singular integral operators $T$ in $m$ $\operatorname{GCZO}(A, s, \eta, \epsilon)$ with kernel satisfying Assumption 4, some authors have done so much work. Duong et al. [5] obtained that $T$ maps $L^{p_{1}}(\omega) \times \cdots \times L^{p_{m}}(\omega)$ to $L^{p}(\omega)$, where $\omega \in A_{p_{0}}$ with $p_{0}=\min \left(p_{1}, \ldots, p_{m}\right)>1$. Grafakos et al. [8] proved that $T$ maps $L^{p_{1}}\left(\omega_{1}\right) \times \cdots \times L^{p_{m}}\left(\omega_{m}\right)$ to $L^{p}\left(\nu_{\vec{\omega}}\right)$ where all $p_{j}>1$ and $\vec{\omega} \in A_{\vec{P}}$, and maps $L^{p_{1}}\left(\omega_{1}\right) \times \cdots \times L^{p_{m}}\left(\omega_{m}\right)$ to $L^{p, \infty}\left(\nu_{\vec{\omega}}\right)$ with some $p_{j}=1$. For $\vec{\omega} \in \prod_{j=1}^{m} A_{p_{j}}$ with $p_{j}>1, j=1, \ldots, m$, Anh and Duong [6] established that $T_{\Sigma \vec{b}}$ are of boundedness from $L^{p_{1}}\left(\omega_{1}\right) \times \cdots \times L^{p_{m}}\left(\omega_{m}\right)$ to $L^{p}\left(\nu_{\vec{\omega}}\right)$; after that, Chen and $\mathrm{Wu}$ [9] extended the results of Lerner et al. [7] and Pérez et al. [2] to the multilinear singular integral operators $T$ in $m$ $\operatorname{GCZO}(A, s, \eta, \epsilon)$ without the endpoint case.
Definition 5. Some multilinear maximal function used in Theorem 6 will be listed in the following, which are introduced by Lerner et al. [7] and Grafakos et al. [8]:

$$
\begin{gathered}
\mathscr{M}(\vec{f})(x)=\sup _{\mathrm{Q} \ni x} \prod_{j=1}^{m} \frac{1}{|Q|} \int_{Q}\left|f_{j}\left(y_{j}\right)\right| d y_{j}, \\
\mathscr{M}_{r}(\vec{f})(x)=\sup _{\mathrm{Q} \ni x} \prod_{j=1}^{m} \frac{1}{|Q|}\left(\int_{Q}\left|f_{j}\left(y_{j}\right)\right|^{r} d y_{j}\right)^{1 / r}, \\
\mathscr{M}_{L \log L}(\vec{f})(x)=\sup _{\mathrm{Q} \ni x} \prod_{j=1}^{m}\left\|f_{j}\right\|_{L \log L, Q^{*}}
\end{gathered}
$$

The following relationship with the above three maximal functions is easy to check:

$$
\mathscr{M}(\vec{f})(x) \leq \mathscr{M}_{L \log L}(\vec{f})(x) \leq C \mathscr{M}_{r}(\vec{f})(x) .
$$

Let $r>1,1 \leq l<m, \sigma=\left\{j_{1}, \ldots, j_{l}\right\} \subset\{1, \ldots, m\}$, and $\sigma^{\prime}=\{1, \ldots, m\} \backslash \sigma$. We define the following multilinear maximal functions:

$$
\begin{aligned}
& \mathscr{M}_{\sigma}(\vec{f})(x) \\
&=\sup _{\mathrm{Q} \ni x_{k=0}} \sum_{k=0}^{\infty} 2^{-k n l} \prod_{j \in \sigma} \frac{1}{|Q|} \int_{Q}\left|f_{j}\left(y_{j}\right)\right| d y_{j} \\
& \quad \times \prod_{j \in \sigma^{\prime}} \frac{1}{\left|2^{k} Q\right|}\left|f_{j}\left(y_{j}\right)\right| d y_{j},
\end{aligned}
$$

$$
\begin{gathered}
\mathscr{M}_{\sigma}(\vec{f})(x) \\
=\sup _{\mathrm{Q} \ni x_{k=0}}^{\infty} 2^{-k n l} \prod_{j \in \sigma}\left(\frac{1}{|Q|} \int_{Q}\left|f_{j}\left(y_{j}\right)\right| d y_{j}\right)^{1 / r} \\
\times \prod_{j \in \sigma^{\prime}}\left(\frac{1}{\left|2^{k} Q\right|}\left|f_{j}\left(y_{j}\right)\right|^{r} d y_{j}\right)^{1 / r}, \\
\mathscr{M}_{\sigma, L \log L}(\vec{f})(x) \\
=\sup _{\mathrm{Q} \ni x} \sum_{k=0}^{\infty} 2^{-k n l} \prod_{j \in \sigma}\left\|f_{j}\right\|_{L \log L, Q} \prod_{j \in \sigma^{\prime}}\left\|f_{j}\right\|_{L \log L, 2^{k} Q} \cdot
\end{gathered}
$$

We have that

$\mathscr{M}_{\sigma}(\vec{f})(x) \leq \mathscr{M}_{\sigma, L \log L}(\vec{f})(x) \leq C \mathscr{M}_{\sigma, r}(\vec{f})(x)$.

The following statements are our main results.

Theorem 6. Let $0<k<1,1 \leq p_{1}, \ldots, p_{m}<\infty, 1 / p=1 / p_{1}+$ $\cdots+1 / p_{m}$, and $\vec{\omega} \in \prod_{i=1}^{m} A_{p_{i}}$ Let $1 \leq j<m, \sigma=\left\{i_{1}, \ldots, i_{j}\right\} \subset$ $\{1, \ldots, m\}$, and for some $r>1$ ( $r$ depending only on $\vec{\omega})$, if all $p_{i}>1$, then $\mathscr{M}_{r}$ and $\mathscr{M}_{\sigma, r}$ are bounded from $L^{p_{1}, k}\left(\omega_{1}\right) \times \cdots \times$ $L^{p_{m}, k}\left(\omega_{m}\right)$ to $L^{p, k}\left(\nu_{\vec{\omega}}\right)$, and or else, bounded from $L^{p_{1}, k}\left(\omega_{1}\right) \times$ $\cdots \times L^{p_{m}, k}\left(\omega_{m}\right)$ to $W L^{p, k}\left(\nu_{\vec{\omega}}\right)$. 
Corollary 7. Under the same assumptions as in Theorem 6. $\mathscr{M}, \mathscr{M}_{L \log L}, \mathscr{M}_{\sigma}, \mathscr{M}_{\sigma, L \log L}$ are bounded from $L^{p_{1}, k}\left(\omega_{1}\right) \times \cdots \times$ $L^{p_{m}, k}\left(\omega_{m}\right)$ to $L^{p, k}\left(\nu_{\vec{\omega}}\right)$ or $W L^{p, k}\left(\nu_{\vec{\omega}}\right)$.

Theorem 8. Assume that $T$ is a multilinear operator in $m$ $\operatorname{GCZO}(A, s, \eta, \epsilon)$ with kernel $K$ satisfying Assumption 4. Let $0<k<1,1 / m \leq p<\infty, 1 \leq p_{j} \leq \infty$ with $1 / p=$ $1 / p_{1}+\cdots+1 / p_{m}$, and $\omega_{j} \in A_{p_{j}}, j=1, \ldots, m$. Then we have the following:

(i) when all $p_{j}>1$, there exists a constant $C$ such that

$$
\|T(\vec{f})\|_{L^{p, k}\left(v_{\vec{\omega}}\right)} \leq C\left\|f_{j}\right\|_{L^{p_{j}, k}\left(\omega_{j}\right)}
$$

(ii) when some $p_{j}=1$, there exists a constant $C$ such that

$$
\|T(\vec{f})\|_{W L^{p, k}\left(v_{\vec{\omega}}\right)} \leq C\left\|f_{j}\right\|_{L^{p_{j}, k}\left(\omega_{j}\right)}
$$

where $\nu_{\vec{\omega}}=\prod_{j=1}^{m} \omega_{j}^{p / p_{j}}$

Theorem 9. Assume that $T$ is a multilinear operator in $m$ $\operatorname{GCZO}(A, s, \eta, \epsilon)$ with kernel $K$ satisfying Assumption 4. Let $0<k<1, \vec{\omega}=\left(\omega_{1}, \ldots, \omega_{m}\right) \in \prod_{j=1}^{m} A_{p_{j}}$, and $\nu_{\vec{\omega}}=\prod_{j=1}^{m} \omega_{j}^{p / p_{j}}$ with $1 / p=1 / p_{1}+\cdots+p_{m}$ and $1<p_{j}<\infty, j=1, \ldots, m$ and $\vec{b}=\left(b_{1}, \ldots, b_{m}\right) \in B M O^{m}$. Then, there exists a constant $C$ such that

$$
\begin{aligned}
& \left\|T_{\Sigma \vec{b}}(\vec{f})\right\|_{L^{p, k}\left(v_{\vec{\omega}}\right)} \leq C\left\|f_{j}\right\|_{L^{p_{j}, k}\left(\omega_{j}\right)^{\prime}} \\
& \left\|T_{\Pi \vec{b}}(\vec{f})\right\|_{L^{p, k}\left(v_{\vec{\omega}}\right)} \leq C\left\|f_{j}\right\|_{L^{p_{j}, k}\left(\omega_{j}\right)} .
\end{aligned}
$$

Following [2], for positive integers $m$ and $j$ with $1 \leq$ $j \leq m$, we denote by $C_{j}^{m}$ the family of all finite subsets $\sigma=$ $\{\sigma(1), \ldots, \sigma(j)\}$ of $\{1, \ldots, m\}$ of $j$ different elements, where we always take $\sigma(l)<\sigma(k)$ if $l<k$. For any $\sigma \in C_{j}^{m}$, the associated complementary sequence $\sigma^{\prime} \in C_{m-j}^{m}$ is given by $\sigma^{\prime}=\{1, \ldots, m\} \backslash \sigma$ with the convention $C_{0}^{m}=\emptyset$. Given an $m$-tuple of functions $\vec{b}$ and $\sigma \in C_{j}^{m}$, we also use the notation $\vec{b}_{\sigma}$ for the $j$-tuple obtained from $\vec{b}$ given by $\left(b_{\sigma(1)}, \ldots, \sigma(j)\right)$. Similar to $T_{\Pi \vec{b}}$, we define for $T$ in $m-G C Z O(A, s, \eta, \epsilon), \sigma \in C_{j}^{m}$ and $\vec{b}_{\sigma}=\left(b_{\sigma(1)}, \ldots, b_{\sigma(j)}\right)$ in $B M O^{j}$, the $j$ th order iterated commutator

$$
T_{\Pi \vec{b}_{\sigma}}(\vec{f})=\left[b_{\sigma(1)},\left[b_{\sigma(2)}, \ldots,\left[b_{\sigma(j)}, T\right]_{\sigma(j)} \ldots\right]_{\sigma(2)}\right]_{\sigma(1)}(\vec{f}) ;
$$

that is, formally

$$
\begin{aligned}
T_{\Pi \vec{b}_{\sigma}}(\vec{f})(x) \int_{\left(\mathbb{R}^{n}\right)^{m}} & \left(\prod_{i=1}^{j}\left(b_{\sigma(i)}(x)-b_{\sigma(i)}\left(y_{\sigma(i)}\right)\right)\right) \\
& \times K\left(x, y_{1}, \ldots, y_{m}\right) \prod_{i=1}^{m} f_{i}\left(y_{i}\right) d \vec{y} .
\end{aligned}
$$

Clearly, $T_{\Pi \vec{b}_{\sigma}}=T_{\Pi \vec{b}}$ when $\sigma=\{1, \ldots, m\}$, and $T_{\Pi \vec{b}_{\sigma}}=T_{b_{j}}^{j}$ when $\sigma=\{j\}$. We have the following general forms of Theorem 9 without the proof.

Theorem 10. Assume that $T$ is a multilinear operator in $m$ $\operatorname{GCZO}(A, s, \eta, \epsilon)$ with kernel $K$ satisfying Assumption 4. Let $1 \leq j \leq m, \sigma \in C_{j}^{m}, \vec{\omega}=\left(\omega_{1}, \ldots, \omega_{m}\right) \in \prod_{i=1}^{m} A_{p_{i}}$, and $\nu_{\vec{\omega}}=\prod_{i=1}^{m} \omega_{i}^{p / p_{i}}$ with $1 / p=1 / p_{1}+\cdots+p_{m}$ and $1<p_{i}<$ $\infty, i=1, \ldots, m$ and $\vec{b}_{\sigma}=\left(b_{\sigma(1)}, \ldots, b_{\sigma(j)}\right) \in B M O^{j}$. Then, there exists a constant $C$ such that

$$
\left\|T_{\Pi \vec{b}_{\sigma}}(\vec{f})\right\|_{L^{p, k}\left(v_{\vec{\omega}}\right)} \leq C \prod_{i=1}^{j}\left\|b_{\sigma(i)}\right\| \prod_{i=1}^{m}\left\|f_{i}\right\|_{L^{p_{i}, k}\left(\omega_{i}\right)} .
$$

\section{Some Definitions and Results}

In this section, we introduce some definitions and results used be later on.

Definition 11 ( $A_{p}$ weights). A weight $\omega$ is a nonnegative, locally integrable function on $\mathbb{R}^{n}$. Let $1<p<\infty$; we call that a weight function $\omega$ that belongs to the class $A_{p}$, if there is a constant $C$ such that, for any cube $Q$,

$$
\left(\frac{1}{|Q|} \int_{Q} \omega(x) d x\right)\left(\frac{1}{|Q|} \int_{Q} \omega(x)^{1-p^{\prime}} d x\right)^{p-1} \leq C,
$$

and $\omega$ belongs to the class $A_{1}$, if there is a constant $C$ such that, for any cube $Q$,

$$
\frac{1}{|Q|} \int_{Q} \omega(x) d x \leq C \inf _{x \in Q} \omega(x)
$$

We denote $A_{\infty}=\cup_{p>1} A_{p}$.

Definition 12 (see [7]). For $m$ exponents $p_{1}, \ldots, p_{m} \in[1, \infty)$, we often write $p$ for the number given by $p=\sum_{j=1}^{m} p_{j}$ and denote $\vec{P}$ by the vector $\vec{P}=\left(p_{1}, \ldots, p_{m}\right)$. A multiple weight $\vec{\omega}=\left(\omega_{1}, \ldots, \omega_{m}\right)$ is said to satisfy the $A_{\vec{P}}$ condition if for

$$
\nu_{\vec{\omega}}=\prod_{j=1}^{m} \omega^{p / p_{j}}
$$

it holds that

$$
\begin{aligned}
& \sup _{Q}\left(\frac{1}{|Q|} \int_{Q} \nu_{\vec{\omega}}(x) d x\right)^{1 / p} \prod_{j=1}^{m}\left(\frac{1}{|Q|} \int_{Q} \omega_{j}(x)^{1-p_{j}^{\prime}} d x\right)^{1 / p_{j}^{\prime}} \\
& \quad<\infty
\end{aligned}
$$

when $p_{j}=1,\left((1 /|Q|) \int_{Q} \omega_{j}(x)^{1-p_{j}^{\prime}} d x\right)^{1 / p_{j}^{\prime}}$ is understood as $\left(\inf _{x} \omega(x)\right)^{-1}$. 
As remarked in [7], $\prod_{j=1}^{m} A_{p_{j}}$ is strictly contained in $A_{\vec{p}}$; moreover, in general $\vec{\omega} \in A_{\vec{P}}$ does not imply $\omega_{j} \in L_{\text {loc }}^{1}$ for any $j$, but instead

$$
\vec{\omega} \in A_{\vec{P}} \Longleftrightarrow\left\{\begin{array}{l}
v_{\vec{\omega}} \in A_{m p}, \\
\omega_{j}^{1-p_{j}^{\prime}} \in A_{m p_{j}^{\prime}}, \quad j=1, \ldots, m,
\end{array}\right.
$$

where the condition $\omega_{j}^{1-p_{j}^{\prime}} \in A_{m p_{j}^{\prime}}$ in the case $p_{j}=1$ is understood as $\omega_{j}^{1 / m} \in A_{1}$.

Definition 13 (see [10]). Let $1 \leq p<\infty, 0<k<1$, and $\omega$ be a weight function on $\mathbb{R}^{n}$. The weighted Morrey space is define by

$$
L^{p, k}(\omega)=\left\{f \in L_{\mathrm{loc}}^{p}:\|f\|_{L^{p, k}(\omega)}<\infty\right\},
$$

where

$$
\|f\|_{L^{p, k}(\omega)}=\sup _{Q}\left(\frac{1}{\omega(Q)^{k}} \int_{Q}|f(x)|^{p} \omega(x)\right)^{1 / p}
$$

The weighted weak Morrey space is defined by

$$
W L^{p, k}(\omega)=\left\{f \text { measurable }:\|f\|_{W L^{p, k}(\omega)}<\infty\right\},
$$

where

$$
\|f\|_{W L^{p, k}(\omega)}=\sup _{Q} \sup _{\lambda>0} \frac{\lambda}{\omega(Q)^{k / p}} \omega(\{x \in Q:|f|(x)>\lambda\})^{1 / p} .
$$

We say that a weight $\omega$ satisfies the doubling condition, denot ing $\omega \in \Delta_{2}$, if there is a constant $C>0$ such that $\omega(2 Q) \leq C \omega(Q)$ holds for any cube $Q$. If $\omega \in A_{p}$ with $1 \leq p<\infty$, we know that $\omega(\lambda Q) \leq \lambda^{n p}[\omega]_{A_{p}} \omega(Q)$ for all $\lambda>1$, then $\omega \in \Delta_{2}$.

Lemma 14 (see [10]). Suppose $\omega \in \Delta_{2}$, then there exists a constant $D>1$ such that

$$
\omega(2 Q) \geq D \omega(Q),
$$

for any cube.

Lemma 15 (see [11]). If $\omega_{j} \in A_{\infty}$, then for any cube $Q$, we have

$$
\int_{Q} \prod_{j=1}^{m} \omega_{j}^{\theta_{j}}(x) d x \geq \prod_{j=1}^{m}\left(\frac{\int_{Q} \omega_{j}(x) d x}{\left[\omega_{j}\right]_{\infty}}\right)^{\theta_{j}},
$$

where $\sum_{j=1}^{m} \theta_{j}=1,0 \leq \theta_{j} \leq 1$.
Lemma 16 (see [12]). Suppose $\omega \in A_{\infty}$, then $\|b\|_{B M O(\omega)} \approx$ $\|b\|_{B M O}$. Here

$$
\begin{gathered}
B M O(\omega)=\left\{b:\|b\|_{B M O(\omega)}\right. \\
\left.=\sup _{\mathrm{Q}} \frac{1}{\omega(\mathrm{Q})} \int_{\mathrm{Q}}\left|b(x)-b_{\mathrm{Q}, \omega}\right| \omega(x) d x<\infty\right\}, \\
b_{\mathrm{Q}, \omega}=\frac{1}{\omega(\mathrm{Q})} \int_{\mathrm{Q}} b(x) \omega(x) d x .
\end{gathered}
$$

From the fact $\left|b_{2^{j} \mathrm{Q}}-b_{\mathrm{Q}}\right| \leq C j\|b\|_{\mathrm{BMO}}$ and Lemma 16, we can deduce that $\left|b_{2^{j}, \omega}-b_{\mathrm{Q}, \omega}\right| \leq C j\|b\|_{\mathrm{BMO}}$.

Lemma 17 (see [8]). Assume that $T$ is a multilinear operator in $m-G C Z O(A, s, \eta, \epsilon)$ with kernel $K$ satisfying Assumption 4. Let $1 / m \leq p<\infty, 1 \leq p_{j} \leq \infty$ with $1 / p=1 / p_{1}+\cdots+1 / p_{m}$ and $\omega_{j} \in A_{p_{j}}, j=1, \ldots, m$. Then we have the following:

(i) T extends to a bounded operators from $L^{p_{1}}\left(\omega_{1}\right) \times \cdots \times$ $L^{p_{m}}\left(\omega_{m}\right)$ to $L^{p}\left(v_{\vec{\omega}}\right)$ if all the exponents $p_{j}$ are strictly greater than 1;

(ii) $T$ extends to a bounded operators from $L^{p_{1}}\left(\omega_{1}\right) \times \cdots \times$ $L^{p_{m}}\left(\omega_{m}\right)$ to $L^{p, \infty}\left(v_{\vec{\omega}}\right)$ if some exponents $p_{j}$ are equal to 1 .

In either case, the norm of $T$ is bounded by $C(A+$ $\left.\|T\|_{L^{q_{1} \times \cdots \times q_{m}}} \rightarrow L^{q}\right)$, where $C$ is a positive constant depending on $A, s, \eta, \epsilon$, and $[w]_{A_{\vec{P}}}$.

Lemma 18 (see [6]). Assume that $T$ is a multilinear operator in $m-G C Z O(A, s, \eta, \epsilon)$ with kernel $K$ satisfying Assumption 4. Let $\vec{b} \in B M O^{m}$ with $\|\vec{b}\|=1$ and $1 / p=1 / p_{1}+\cdots 1 / p_{m}$ with $1<p_{j}<\infty, j=1, \ldots, m$. Then we have the following:

(i) there exists a constant $C$ such that

$$
\left\|T_{\Sigma \vec{b}}(\vec{f})\right\|_{L^{p}\left(v_{\vec{\omega}}\right)} \leq C \prod_{j=1}^{m}\left\|f_{j}\right\|_{L^{p_{j}}\left(M \omega_{j}\right)}
$$

(ii) if $\omega_{j} \in A_{p_{j}}$, then there exists a constant $C$ such that

$$
\left\|T_{\Sigma \vec{b}}(\vec{f})\right\|_{L^{p}\left(v_{\vec{\omega}}\right)} \leq C \prod_{j=1}^{m}\left\|f_{j}\right\|_{L^{p_{j}}\left(\omega_{j}\right)}
$$

where $v_{\vec{\omega}}=\prod_{j=1}^{m} \omega_{j}^{p / p_{j}}$.

Lemma 19 (see [9]). Assume that $T$ is a multilinear operator in $m-G C Z O(A, s, \eta, \epsilon)$ with kernel $K$ satisfying Assumption 4. Let $\vec{b} \in B M O^{m}$ with $\|\vec{b}\|=1$ and $1 / p=1 / p_{1}+\cdots 1 / p_{m}$ with $1<p_{j}<\infty, j=1, \ldots, m$. If $\omega_{j} \in A_{\vec{P}}$ with $\vec{P}=\left(p_{1}, \ldots, p_{m}\right)$, then there exists a constant $C$ such that

$$
\left\|T_{\Pi \vec{b}}(\vec{f})\right\|_{L^{p}\left(\nu_{\vec{\omega}}\right)} \leq C \prod_{j=1}^{m}\left\|f_{j}\right\|_{L^{p_{j}}\left(\omega_{j}\right)}
$$

where $v_{\vec{\omega}}=\prod_{j=1}^{m} \omega_{j}^{p / p_{j}}$. 


\section{Proof of Theorems}

Proof of Theorem 6. Here, we only prove the boundedness of $\mathscr{M}_{\sigma, r}$. From [9], there exists some $t \in(0,1)$ only depend on $\vec{\omega}$ such that

$$
\mathscr{M}(\vec{f})(x) \leq C \prod_{j=1}^{m}\left\{M_{\nu_{\vec{\omega}}}^{c}\left(\left(\left|f_{j}\right|^{p_{j}} \omega_{j} / \nu_{\vec{\omega}}\right)^{t}\right)(x)\right\}^{1 / t p_{j}},
$$

where $M_{\nu_{\tilde{\omega}}}^{c}$ is the weighted centered maximal operator. Then by the Hölder inequality,

$$
\begin{aligned}
& \left\|M_{\sigma, r}(\vec{f})(x)\right\|_{L^{p, k}\left(v_{\vec{\omega}}\right)} \\
& \leq C\left\|\prod_{i=1}^{m}\left\{M_{v_{\vec{\omega}}}\left(\left[\left|f_{i}\right|^{p_{i}} \omega_{i} / v_{\vec{\omega}}\right]^{t}\right)\right\}^{1 / t p_{i}}\right\|_{L^{p, k}\left(v_{\vec{\omega}}\right)} \\
& \leq C \prod_{i=1}^{m}\left\|\left\{M_{v_{\vec{\omega}}}\left(\left[\left|f_{i}\right|^{p_{i}} \omega_{i} / \nu_{\vec{\omega}}\right]^{t}\right)\right\}^{1 / t p_{i}}\right\|_{L^{p_{i}, k}\left(v_{\vec{\omega}}\right)} \\
& \leq C \prod_{i=1}^{m}\left\|\left\{M_{v_{\vec{\omega}}}\left(\left[\left|f_{i}\right|^{p_{i}} \omega_{i} / \nu_{\vec{\omega}}\right]^{t}\right)\right\}^{1 / t p_{i}}\right\|_{L^{1 / t, k}\left(v_{\vec{\omega}}\right)}^{1 / t p_{i}} \\
& \leq C \prod_{i=1}^{m}\left\|\left(\left|f_{i}\right|^{p_{i}} \omega_{i} / v_{\vec{\omega}}\right)^{t}\right\|_{L^{1 / t, k}\left(v_{\vec{\omega}}\right)}^{1 / t p_{i}} \\
& \leq C \prod_{i=1}^{m}\left\|f_{i}\right\|_{L^{p_{i}, k}\left(\omega_{i}\right)} .
\end{aligned}
$$

The weak version is a very similar process by the Hölder inequality for the weak spaces. We omit the details.

Proof of Theorem 8. For any $B=B\left(x_{B}, r_{B}\right) \subset \mathbb{R}^{n}$, we split $f_{i}=$ $f_{i}^{0}+f_{i}^{\infty}$ where $f_{i}^{0}=f_{i} \chi_{B^{*}}, i=1,2, \ldots, m$, and $B^{*}=8 B$; then

$$
\begin{aligned}
\prod_{i=1}^{m} f_{i}\left(y_{i}\right) & =\prod_{i=1}^{m}\left(f_{i}^{0}\left(y_{i}\right)+f_{i}^{\infty}\left(y_{i}\right)\right) \\
& =\sum_{\alpha_{1}, \ldots, \alpha_{m} \in\{0, \infty\}} \prod_{i=1}^{m} f_{i}^{\alpha_{i}}\left(y_{i}\right) \\
& =\prod_{i=1}^{m} f_{i}^{0}\left(y_{i}\right)+\sum^{\prime} f_{1}^{\alpha_{1}}\left(y_{1}\right) \cdots f_{m}^{\alpha_{m}}\left(y_{m}\right),
\end{aligned}
$$

where each term of $\sum^{\prime}$ contains at least one $\alpha_{i} \neq 0$. Write then

$$
\begin{aligned}
& \frac{1}{v_{\vec{\omega}}(B)^{k / p}}\left(\int_{B}\left|T\left(f_{1}, \ldots, f_{m}\right)(x)\right|^{p} v_{\vec{\omega}}(x) d x\right)^{1 / p} \\
& \leq \frac{1}{v_{\vec{\omega}}(B)^{k / p}}\left(\int_{B}\left|T\left(f_{1}^{0}, \ldots, f_{m}^{0}\right)(x)\right|^{p} v_{\vec{\omega}}(x) d x\right)^{1 / p} \\
& \quad+\sum^{\prime} \frac{1}{v_{\vec{\omega}}(B)^{k / p}}\left(\int_{B}\left|T\left(f_{1}^{\alpha_{1}}, \ldots, f_{m}^{\alpha_{m}}\right)(x)\right|^{p} v_{\vec{\omega}}(x) d x\right)^{1 / p} \\
& =I^{0, \ldots, 0}+\sum^{1} I^{\alpha_{1}, \ldots, \alpha_{m}} .
\end{aligned}
$$

From Definition 12, Lemma 17, we can get

$$
\begin{aligned}
I^{0, \ldots, 0} & \leq \frac{C}{v_{\vec{\omega}}(B)^{k / p}} \prod_{i=1}^{m}\left(\int_{B^{*}}\left|f_{i}^{0}(x)\right|^{p_{i}} \omega_{i}(x) d x\right)^{1 / p_{i}} \\
& \leq C \frac{\prod_{i=1}^{m} \omega_{i}\left(B^{*}\right)^{k / p}}{v_{\vec{\omega}}(B)^{k / p}} \prod_{i=1}^{m}\left\|f_{i}\right\|_{L^{p, k}\left(\omega_{i}\right)} \\
& \leq C \prod_{i=1}^{m}\left\|f_{i}\right\|_{L^{p, k}\left(\omega_{i}\right)} .
\end{aligned}
$$

The last inequality holds by Lemma 15 . For $\sum^{\prime} I^{\alpha_{1}, \ldots, \alpha_{m}}$, we first consider the case when $\alpha_{1}=\cdots=\alpha_{m}=\infty$. Taking $t=\left(2 r_{B}\right)^{s}$, since $x \in B$ and $y_{i} \in \mathbb{R}^{n} \backslash 8 B$, we get

$$
\left|y_{i}-x\right|>7 r_{B}>2 t^{1 / s}, \quad \text { for all } j=1, \ldots, m ;
$$

hence, $h\left(\left|y_{i}-x\right| / t^{1 / s}\right)=0$. By Assumption 4, we have that

$$
\begin{aligned}
& \left|K\left(x, y_{1}, \ldots, y_{m}\right)-K_{t}^{(0)}\left(x, y_{1}, \ldots, y_{m}\right)\right| \\
& \quad \leq \frac{A t^{\epsilon / t}}{\left(\sum_{k=1}^{m}\left|x-y_{k}\right|\right)^{m n+\epsilon}} \leq \frac{A}{\left(\sum_{k=1}^{m}\left|x-y_{k}\right|\right)^{m n}} .
\end{aligned}
$$

For any $x \in B$, then by Assumption 4,

$$
\begin{aligned}
& \mid T\left(f_{1}^{\infty}, \ldots, f_{m}^{\infty}\right)(x) \mid \\
& \leq \int_{\left(\mathbb{R}^{n} \backslash B^{*}\right)^{m}}\left|K\left(x, y_{1}, \ldots, y_{m}\right)-K_{t}^{(0)}\left(x, y_{1}, \ldots, y_{m}\right)\right| \\
& \times \prod_{i=1}^{m}\left|f_{i}^{\infty}\left(y_{i}\right)\right| d \vec{y} \\
&+\int_{\left(\mathbb{R}^{n} \backslash B^{*}\right)^{m}}\left|K_{t}^{(0)}\left(x, y_{1}, \ldots, y_{m}\right)\right| \prod_{i=1}^{m}\left|f_{i}^{\infty}\left(y_{i}\right)\right| d \vec{y} \\
& \leq C \int_{\left(\mathbb{R}^{n} \backslash B^{*}\right)^{m}} \frac{A}{\left(\left|x-y_{1}\right|+\cdots+\left|x-y_{m}\right|\right)^{m n}} \\
& \leq C \sum_{l=1}^{\infty} \prod_{i=1}^{m} \int_{8^{l+1} B \mid 8^{j} B} \frac{\left|f_{i}\left(y_{i}\right)\right|}{\left|x-y_{i}\right|^{n}} d y_{i} \\
& \leq C \sum_{l=1}^{\infty} \prod_{i=1}^{m} \frac{1}{\left|8^{l+1} B\right|}\left(\int_{8^{l+1} B}\left|f_{i}^{\infty}\left(y_{i}\right)\right|^{p_{i}} \omega_{i}\left(y_{i}\right) d y_{i}\right)^{1 / p_{i}} \\
& \quad \times\left(\int_{8^{l+1} B} \omega_{i}\left(y_{i}\right)^{1-p_{i}^{\prime}} d y_{i}\right)^{1 / p_{i}^{\prime}} \\
& \leq C \sum_{l=1}^{\infty} \prod_{i=1}^{m} \frac{\omega_{i}\left(8^{l+1} B\right)^{k / p_{i}}}{\left|8^{l+1} B\right|}\left\|f_{i}\right\|_{L^{p_{i}, k}\left(\omega_{i}\right)} \frac{\left|8^{l+1} B\right|}{\omega_{i}\left(8^{l+1} B\right)^{1 / p_{i}}} \\
& \leq \sum_{l=1}^{\infty} v_{\vec{\omega}}\left(8^{l+1} B\right)^{(k-1) / p} \prod_{i=1}^{m}\left\|f_{i}\right\|_{L^{p_{i}, k}\left(\omega_{i}\right)^{\prime}}
\end{aligned}
$$


Since $\nu_{\vec{\omega}} \in A_{m p}$, then there is a positive $\delta$ such that

$$
\frac{\nu_{\omega}(B)}{\nu_{\omega}\left(8^{l+1} B\right)} \leq C\left(\frac{|B|}{\left|8^{l+1} B\right|}\right)^{\delta} .
$$

Hence

$$
\begin{aligned}
I^{\infty, \ldots, \infty} & \leq \nu_{\vec{\omega}}(B)^{(1-k) / p} \sup _{x \in B}\left|T\left(f_{1}^{\infty}, \ldots, f_{m}^{\infty}\right)(x)\right| \\
& \leq C \sum_{l=1}^{\infty}\left(\frac{|B|}{\left|8^{l+1} B\right|}\right)^{\delta(1-k) / p} \prod_{i=1}^{m}\left\|f_{i}\right\|_{L^{p_{i}, k}\left(\omega_{i}\right)} \\
& \leq C \prod_{i=1}^{m}\left\|f_{i}\right\|_{L^{p_{i}, k}\left(\omega_{i}\right)} .
\end{aligned}
$$

It remains to estimate the terms with $\alpha_{i_{1}}=\cdots=\alpha_{i_{j}}=0$ for some $\left\{i_{1}, \ldots, i_{j}\right\} \subset\{1, \ldots, m\}$ and $1 \leq j<m$. We have

$$
\begin{aligned}
& \left|T\left(f_{1}^{\alpha_{1}}, \ldots, f_{m}^{\alpha_{m}}\right)(x)\right| \\
& \leq \int_{\left(\mathbb{R}^{n} \backslash B^{*}\right)^{m}} \mid K\left(x, y_{1}, \ldots, y_{m}\right) \\
& -K_{t}^{(0)}\left(x, y_{1}, \ldots, y_{m}\right)\left|\prod_{i=1}^{m}\right| f_{i}^{\alpha_{i}}\left(y_{i}\right) \mid d \vec{y} \\
& +\int_{\left(\mathbb{R}^{n} \backslash B^{*}\right)^{m}}\left|K_{t}^{(0)}\left(x, y_{1}, \ldots, y_{m}\right)\right| \prod_{i=1}^{m}\left|f_{i}^{\alpha_{i}}\left(y_{i}\right)\right| d \vec{y} \\
& \leq C \prod_{i \in\left\{i_{1}, \ldots, i_{j}\right\}} \int_{B^{*}}\left|f_{i}\left(y_{i}\right)\right| d y_{i} \\
& \times\left[\int_{\left(\mathbb{R}^{n} \backslash B^{*}\right)^{m-j}} \frac{t^{\epsilon / s} \prod_{i \notin\left\{i_{1}, \ldots, i_{j}\right\}}\left|f_{i}\left(y_{i}\right)\right| d y_{i}}{\left(\sum_{i \notin\left\{i_{1}, \ldots, i_{j}\right\}}\left|x-y_{i}\right|\right)^{m n+\epsilon}}\right. \\
& \left.+\int_{\left(\mathbb{R}^{n} \backslash B^{*}\right)^{m-j}} \frac{\prod_{i \notin\left\{i_{1}, \ldots, i_{j}\right\}}\left|f_{i}\left(y_{i}\right)\right| d y_{i}}{\left(\sum_{i \notin\left\{i_{1}, \ldots, i_{j}\right\}}\left|x-y_{i}\right|\right)^{m n}}\right] \\
& \leq C \frac{1}{\left|8^{j+1} B\right|^{m}} \prod_{i \in\left\{i_{1}, \ldots, i_{j}\right\}} \int_{B^{*}}\left|f_{i}\left(y_{i}\right)\right| d y_{i} \\
& \times \sum_{l=1}^{\infty} \prod_{i \notin\left\{i_{1}, \ldots, i_{j}\right\}} \int_{8^{j+1} B \backslash 8^{j} B}\left|f_{i}\left(y_{i}\right)\right| d y_{i} \\
& \leq C \sum_{l=1}^{\infty} \nu_{\vec{\omega}}\left(8^{l+1} B\right)^{(k-1) / p} \prod_{i=1}^{m}\left\|f_{i}\right\|_{L^{p_{i}, k}\left(\omega_{i}\right)}
\end{aligned}
$$

Therefore, we also have

$$
I^{\alpha_{1}, \ldots, \alpha_{m}} \leq C \prod_{i=1}^{m}\left\|f_{i}\right\|_{L^{p_{i}, k}\left(\omega_{i}\right)}
$$

Combining the above estimates and then taking the supermum over all balls $B$ in $\mathbb{R}^{n}$, we have proved the previous part of Theorem 8 .
Next, we turn to complete the proof of the weak inequality. For any $\lambda>0$, we can write

$$
\begin{aligned}
\nu_{\vec{\omega}}\left(\left\{x \in B:\left|T\left(f_{1}, \ldots, f_{m}\right)(x)\right|>\lambda\right\}\right)^{1 / p} \\
\leq \nu_{\vec{\omega}}\left(\left\{x \in B:\left|T\left(f_{1}^{\infty}, \ldots, f_{m}^{\infty}\right)(x)\right|>\lambda\right\}\right)^{1 / p} \\
\quad+\sum^{\prime} \nu_{\vec{\omega}}\left(\left\{x \in B:\left|T\left(f_{1}^{\alpha_{1}}, \ldots, f_{m}^{\alpha_{m}}\right)(x)\right|>\lambda\right\}\right)^{1 / p} \\
=I I^{0, \ldots, 0}+\sum^{\prime} I I^{\alpha_{1}, \ldots, \alpha_{m}} .
\end{aligned}
$$

By Lemmas 17 and 15, we can easily check that

$$
\begin{aligned}
I I^{0, \ldots, 0} & \leq \frac{C}{\lambda} \prod_{i=1}^{m}\left(\int_{B^{*}} f_{i}^{0}\left(y_{i}\right)^{p_{i}} \omega_{i}\left(y_{i}\right) d y_{i}\right)^{1 / p_{i}} \\
& \leq \frac{C \nu_{\vec{\omega}}(B)^{k / p}}{\lambda} \prod_{i=1}^{m}\left\|f_{i}\right\|_{L^{p_{i}, k}\left(\omega_{i}\right)} .
\end{aligned}
$$

From the proof of (53) and (56), we have the following pointwise estimate:

$$
\left|T\left(f_{1}^{\alpha_{1}}, \ldots, f_{m}^{\alpha_{m}}\right)(x)\right| \leq C \sum_{l=1}^{\infty} \prod_{i=1}^{m} \frac{1}{\left|8^{l+1} B\right|} \int_{8^{l+1} B}\left|f_{i}\left(y_{i}\right)\right| d y_{i}
$$

Since at least one $p_{i}=1$, we can assume that $\left\{i_{1}, \ldots, i_{j}\right\} \subset$ $\{1, \ldots, m\}$ such that $p_{i_{1}}=\cdots=p_{i_{j}}=1$ and others greater than 1 . Then,

$$
\begin{aligned}
& \left|T\left(f_{1}^{\alpha_{1}}, \ldots, f_{m}^{\alpha_{m}}\right)(x)\right| \\
& \leq C \sum_{l=1_{i \in\left\{i_{1}, \ldots, i_{j}\right\}}}^{\infty} \frac{1}{\left|8^{l+1} B\right|} \int_{8^{l+1} B}\left|f_{i}\left(y_{i}\right)\right| \omega_{i}\left(y_{i}\right) d y_{i} \\
& \times\left(\inf _{y_{i} \in 8^{l+1} B} \omega_{i}\left(y_{i}\right)\right)^{-1} \\
& \quad \times \prod_{i \notin\left\{i_{1}, \ldots, i_{j}\right\}} \frac{1}{\left|8^{l+1} B\right|}\left(\int_{8^{l+1} B}\left|f_{i}\left(y_{i}\right)\right|^{p_{i}} \omega_{i}\left(y_{i}\right) d y_{i}\right)^{1 / p_{i}} \\
& \left.\times \frac{C}{v_{\vec{\omega}}(B)^{(1-k) / p}} \prod_{i=1}^{m}\left\|f_{i}\right\|_{L^{p_{i}, k}\left(\omega_{i}\right)^{\prime}} \omega_{i}\left(y_{i}\right)^{1-p_{i}^{\prime}} d y_{i}\right)^{1 / p_{i}^{\prime}}
\end{aligned}
$$

Suppose that $\left\{x \in B:\left|T\left(f_{1}^{\alpha_{1}}, \ldots, f_{m}^{\alpha_{m}}\right)(x)\right|>\lambda\right\} \neq \emptyset$; then we have that

$$
\nu_{\vec{\omega}}(B)^{1 / p} \leq \frac{C \nu_{\vec{\omega}}(B)^{k / p}}{\lambda} \prod_{i=1}^{m}\left\|f_{i}\right\|_{L^{p_{i}, k}\left(\omega_{i}\right)}
$$


therefore,

$$
I I^{\alpha_{1}, \ldots, \alpha_{m}} \leq \nu_{\vec{\omega}}(B)^{1 / p} \leq \frac{C v_{\vec{\omega}}(B)^{k / p}}{\lambda} \prod_{i=1}^{m}\left\|f_{i}\right\|_{L^{p_{i}, k}\left(\omega_{i}\right)} .
$$

Taking the supremum over all balls $B \subset \mathbb{R}^{n}$ and all $\lambda>0$, we complete the proof of Theorem 6 .

Proof of Theorem 9. We will show the proof for $T_{\Pi \vec{b}}$ because the proof for $T_{\Sigma \vec{b}}$ is very similar but easier. Moreover, for simplicity of the expansion, we only present the case $m=2$.

For any cube $B$, we also split $f_{i}$ as $f_{i}=f_{i}^{0}+f_{i}^{\infty}$ with $f_{i}^{0}=f_{i} \chi_{B^{\star}}$ and $f_{i}^{\infty}=f_{i}-f_{i}^{0}$. Then it remains only to verify the following inequalities:

$$
\begin{aligned}
I & =\left(\frac{1}{v_{\vec{\omega}}(Q)^{k}} \int_{\mathrm{Q}}\left|T_{\Pi \mathbf{b}}\left(f_{1}^{0}, f_{2}^{0}\right)(x)\right|^{p} \nu_{\vec{\omega}}(x) d x\right)^{1 / p} \\
& \leq C \prod_{j=1}^{2}\left\|b_{j}\right\|_{\mathrm{BMO}} \prod_{j=1}^{2}\left\|f_{j}\right\|_{L^{p_{j}, k}\left(\omega_{j}\right)^{\prime}} \\
I I & =\left(\frac{1}{v_{\vec{\omega}}(Q)^{k}} \int_{\mathrm{Q}}\left|T_{\Pi \mathbf{b}}\left(f_{1}^{0}, f_{2}^{\infty}\right)(x)\right|^{p} \nu_{\vec{\omega}}(x) d x\right)^{1 / p} \\
& \leq C \prod_{j=1}^{2}\left\|b_{j}\right\|_{\mathrm{BMO}} \prod_{j=1}^{2}\left\|f_{j}\right\|_{L^{p_{j}, k}\left(\omega_{j}\right)^{\prime}} \\
I I I & =\left(\frac{1}{v_{\vec{\omega}}(Q)^{k}} \int_{\mathrm{Q}}\left|T_{\Pi \mathbf{b}}\left(f_{1}^{\infty}, f_{2}^{0}\right)(x)\right|^{p} \nu_{\vec{\omega}}(x) d x\right)^{1 / p} \\
& \leq C \prod_{j=1}^{2}\left\|b_{j}\right\|_{\mathrm{BMO}} \prod_{j=1}^{2}\left\|f_{j}\right\|_{L^{p_{j}, k}\left(\omega_{j}\right)^{\prime}} \\
& \leq C \prod_{j=1}^{2}\left\|b_{j}\right\|_{\mathrm{BMO}} \prod_{j=1}^{2}\left\|f_{j}\right\|_{L^{p_{j}, k}\left(\omega_{j}\right)^{\cdot}} \\
I V & =\left(\frac{1}{v_{\vec{\omega}}(Q)^{k}} \int_{\mathrm{Q}}\left|T_{\Pi \mathbf{b}}\left(f_{1}^{\infty}, f_{2}^{\infty}\right)(x)\right|^{p} \nu_{\vec{\omega}}(x) d x\right)^{1 / p}
\end{aligned}
$$

From Lemma 19, Lemma 15, and Hölder's inequality, we can get

$$
\begin{aligned}
I & \leq C \frac{1}{v_{\vec{\omega}}(Q)^{k / p}} \prod_{j=1}^{2}\left\|b_{j}\right\|_{\mathrm{BMO}}\left(\int_{\mathbb{R}^{n}}\left|f_{j}^{0}(x)\right|^{p_{j}} \omega_{j}(x) d x\right)^{1 / p_{j}} \\
& \leq C \frac{1}{\nu_{\vec{\omega}}(Q)^{k / p}} \prod_{j=1}^{2}\left[\left\|b_{j}\right\|_{\mathrm{BMO}} \omega_{j}(2 Q)^{k / p_{j}}\left\|f_{j}\right\|_{L^{p_{j}, k}\left(\omega_{j}\right)}\right] \\
& \leq C \prod_{j=1}^{2}\left[\left\|b_{j}\right\|_{\mathrm{BMO}}\left\|f_{j}\right\|_{L^{p_{j}, k}\left(\omega_{j}\right)}\right] .
\end{aligned}
$$

Since $I I$ and $I I I$ are symmetric, we only estimate $I I$. Taking $\lambda_{j}=\left(b_{j}\right)_{B, \omega_{j}}, T_{\Pi \vec{b}}$ can be divided into four part:

$$
\begin{aligned}
T_{\Pi \mathbf{b}} & \left(f_{1}^{0}, f_{2}^{\infty}\right)(x) \\
= & \left(b_{1}(x)-\lambda_{1}\right)\left(b_{2}(x)-\lambda_{2}\right) T\left(f_{1}^{0}, f_{2}^{\infty}\right)(x) \\
& -\left(b_{1}(x)-\lambda_{1}\right) T\left(f_{1}^{0},\left(b_{2}-\lambda_{2}\right) f_{2}^{\infty}\right)(x) \\
& -\left(b_{2}(x)-\lambda_{2}\right) T\left(\left(b_{1}-\lambda_{1}\right) f_{1}^{0}, f_{2}^{\infty}\right)(x) \\
& +T\left(\left(b_{1}-\lambda_{1}\right) f_{1}^{0},\left(b_{2}-\lambda_{2}\right) f_{2}^{\infty}\right)(x) \\
= & I I_{1}+I I_{2}+I I_{3}+I I_{4} .
\end{aligned}
$$

From the proof of Theorem 8 we know that, for any $x \in B$,

$$
\left|T\left(f_{1}^{0}, f_{2}^{\infty}\right)(x)\right| \leq C \sum_{l=1}^{\infty} \nu_{\vec{\omega}}\left(8^{l+1} B\right)^{(k-1) / p} \prod_{i=1}^{m}\left\|f_{j}\right\|_{L^{p_{j}, k}\left(\omega_{j}\right)}
$$

Applying (67), Hölder's inequality and Lemma 16, we have

$$
\begin{aligned}
& \left(\frac{1}{v_{\vec{\omega}}(Q)^{k}} \int_{Q}\left|I I_{1}\right|^{p} \nu_{\vec{\omega}}(x) d x\right)^{1 / p} \\
& \leq \frac{1}{v_{\vec{\omega}}(Q)^{k / p}}\left(\int_{Q}\left|\left(b_{1}(x)-\lambda_{1}\right)\left(b_{2}(x)-\lambda_{2}\right)\right|^{p}\right. \\
& \left.\quad \times v_{\vec{\omega}}(x) d x\right)^{1 / p} \\
& \quad \times \prod_{j=1}^{2}\left\|f_{j}\right\|_{L^{p_{j}, k}} \sum_{l=1}^{\infty} v_{\vec{\omega}}\left(2^{l+1} Q\right)^{(k-1) / p} \\
& \leq \frac{v_{\vec{\omega}}(Q)^{1 / p}}{v_{\vec{\omega}}(Q)^{k / p}} \prod_{j=1}^{2}\left(\frac{1}{\nu_{\vec{\omega}}(Q)} \int_{Q}\left|\left(b_{j}(x)-\lambda_{1}\right)\right|^{2 p} \nu_{\vec{\omega}}(x) d x\right)^{1 / 2 p} \\
& \quad \times \prod_{j=1}^{2}\left\|f_{j}\right\|_{L^{p_{j}, k}} \sum_{l=1}^{\infty} v_{\vec{\omega}}\left(2^{l+1} Q\right)^{(k-1) / p} \\
& \leq \prod_{j=1}^{2}\left\|b_{j}\right\|_{\mathrm{BMO}}\left\|f_{j}\right\|_{L^{p_{j}, k}\left(\omega_{j}\right)}
\end{aligned}
$$

The last inequality is obtained by the property of $A_{\infty}$ : there is a constant $\delta>0$ such that

$$
\frac{\nu_{\vec{\omega}}(Q)}{\nu_{\vec{\omega}}\left(2^{l+1} Q\right)} \leq C\left(\frac{|Q|}{\left|2^{l+1} Q\right|}\right)^{\delta}
$$


For $I_{2}$, by the Assumption 4, Lemma 15, and Lemma 16, it follows that

$$
\begin{aligned}
& \left|T\left(f_{1}^{0},\left(b_{2}-\lambda_{2}\right) f_{2}^{\infty}\right)(x)\right| \\
& \leq \int_{\left(\mathbb{R}^{n}\right)^{2}}\left|K\left(x, y_{1}, y_{2}\right)-K_{t}^{(0)}\left(x, y_{1}, y_{2}\right)\right| \\
& \times\left|f_{1}^{0}\left(y_{1}\right)\left(b_{2}\left(y_{2}\right)-\lambda_{2}\right) f_{2}^{\infty}\left(y_{2}\right)\right| d y_{1} d y_{2} \\
& +\int_{\left(\mathbb{R}^{n}\right)^{2}}\left|K_{t}^{(0)}\left(x, y_{1}, y_{2}\right)\right| \mid f_{1}^{0}\left(y_{1}\right)\left(b_{2}\left(y_{2}\right)-\lambda_{2}\right) \\
& \times f_{2}^{\infty}\left(y_{2}\right) \mid d y_{1} d y_{2} \\
& \leq C \int_{8 B}\left|f_{1}\left(y_{1}\right)\right| d y_{1} \sum_{l=1}^{\infty} \frac{1}{\left|8^{l} B\right|^{2}} \\
& \times \int_{2^{l+1} Q \mid 2^{l} Q}\left|\left(b_{2}\left(y_{2}\right)-\lambda_{2}\right) f_{2}\left(y_{2}\right)\right| d y_{2} \\
& \leq C \sum_{l=1}^{\infty} \frac{\left\|b_{2}\right\|_{\mathrm{BMO}}}{\left|8^{l+1} B\right|^{2}}\left(\int_{8^{l+1} B}\left|f_{1}\left(y_{1}\right)\right|^{p_{1}} \omega_{j}\left(y_{1}\right) d y_{1}\right)^{1 / p_{1}} \\
& \times\left(\int_{2^{l+1} \mathrm{Q}} \omega_{1}\left(y_{1}\right)^{1-p_{1}^{\prime}} d y_{j}\right)^{1 / p_{1}^{\prime}} \\
& \times\left(\int_{2^{l+1} Q}\left|f_{2}\left(y_{2}\right)\right|^{p_{2}} \omega_{2}\left(y_{2}\right) d y_{2}\right)^{1 / p_{2}} \\
& \times\left(\int_{2^{l+1} Q}\left|b_{2}\left(y_{2}\right)-\lambda_{2}\right|^{p_{2}^{\prime}} \omega_{2}\left(y_{2}\right)^{-p_{2}^{\prime} / p_{2}} d y_{2}\right)^{1 / p_{2}^{\prime}} \\
& \leq C \sum_{l=1}^{\infty} l \prod_{j=1}^{2} \frac{\left\|b_{2}\right\|_{\mathrm{BMO}}}{\left|8^{l+1} B\right|}\left(\int_{8^{l+1} B}\left|f_{j}\left(y_{j}\right)\right|^{p_{j}} \omega_{j}\left(y_{j}\right) d y_{j}\right)^{1 / p_{j}} \\
& \times\left(\int_{2^{l+1} \mathrm{Q}} \omega_{j}\left(y_{j}\right)^{1-p_{j}^{\prime}} d y_{j}\right)^{1 / p_{j}^{\prime}} \\
& \leq C\left\|b_{2}\right\|_{\mathrm{BMO}} \prod_{j=1}^{2}\left\|f_{j}\right\|_{L^{p_{j}, k}\left(\omega_{j}\right)} \sum_{l=1}^{\infty} l \nu_{\vec{\omega}}\left(8^{l+1} B\right)^{(k-1) / p} .
\end{aligned}
$$

Hölder's inequality and Lemma 16 tell us that

$$
\begin{aligned}
& \left(\frac{1}{\nu_{\vec{\omega}}(Q)^{k}} \int_{Q}\left|I I_{2}\right|^{p} \nu_{\vec{\omega}}(x) d x\right)^{1 / p} \\
& \leq C \frac{1}{\nu_{\vec{\omega}}(Q)^{k / p}}\left(\int_{Q}\left|b_{1}(x)-\lambda_{1}\right|^{p} \nu_{\vec{\omega}}(x) d x\right)^{1 / p} \\
& \quad \times \prod_{j=1}^{2}\left\|f_{j}\right\|_{L^{p_{j}, k}} \sum_{l=1}^{\infty} l \nu_{\vec{\omega}}\left(2^{l+1} Q\right)^{(k-1) / p}
\end{aligned}
$$

$$
\begin{aligned}
& \leq C \frac{\nu_{\vec{\omega}}(Q)^{1 / p}}{\nu_{\vec{\omega}}(Q)^{k / p}} \prod_{j=1}^{2}\left\|f_{j}\right\|_{L^{p_{j}, k}} \sum_{l=1}^{\infty} l \nu_{\vec{\omega}}\left(2^{l+1} Q\right)^{(k-1) / p} \\
& \leq C \prod_{j=1}^{2}\left\|b_{j}\right\|_{\mathrm{BMO}}\left\|f_{j}\right\|_{L^{p_{j}, k}\left(\omega_{j}\right)} .
\end{aligned}
$$

Similarly, we also have that

$$
\left(\frac{1}{\nu_{\vec{\omega}}(Q)^{k}} \int_{Q}\left|I I_{3}\right|^{p} \nu_{\vec{\omega}}(x) d x\right)^{1 / p} \leq C \prod_{j=1}^{2}\left\|b_{j}\right\|_{\mathrm{BMO}}\left\|f_{j}\right\|_{L^{p_{j}, k}\left(\omega_{j}\right)} .
$$

By Assumption 4, Lemma 15, and Lemma 16, a similar way deduces that

$$
\begin{aligned}
& \left|T\left(\left(b_{1}-\lambda_{1}\right) f_{1}^{0},\left(b_{2}-\lambda_{2}\right) f_{2}^{\infty}\right)(x)\right| \\
& \quad \leq C\left\|b_{1}\right\|_{\mathrm{BMO}}\left\|b_{2}\right\|_{\mathrm{BMO}} \prod_{j=1}^{2}\left\|f_{j}\right\|_{L^{p_{j}, k}\left(\omega_{j}\right)} \sum_{l=1}^{\infty} l^{2} \nu_{\vec{\omega}}\left(8^{l+1} B\right)^{(k-1) / p},
\end{aligned}
$$

and so,

$$
\left(\frac{1}{\nu_{\vec{\omega}}(Q)^{k}} \int_{Q}\left|I I_{4}\right|^{p} \nu_{\vec{\omega}}(x) d x\right)^{1 / p} \leq C \prod_{j=1}^{2}\left\|b_{j}\right\|_{\mathrm{BMO}}\left\|f_{j}\right\|_{L^{p_{j}, k}\left(\omega_{j}\right)} .
$$

Finally, we still decompose $T_{\Pi \mathbf{b}}\left(f_{1}^{\infty}, f_{2}^{\infty}\right)(x)$ into four terms:

$$
\begin{aligned}
T_{\text {П }}( & \left.f_{1}^{\infty}, f_{2}^{\infty}\right)(x) \\
= & \left(b_{1}(x)-\lambda_{1}\right)\left(b_{2}(x)-\lambda_{2}\right) T\left(f_{1}^{\infty}, f_{2}^{\infty}\right)(x) \\
& -\left(b_{1}(x)-\lambda_{1}\right) T\left(f_{1}^{\infty},\left(b_{2}-\lambda_{2}\right) f_{2}^{\infty}\right)(x) \\
& -\left(b_{2}(x)-\lambda_{2}\right) T\left(\left(b_{1}-\lambda_{1}\right) f_{1}^{\infty}, f_{2}^{\infty}\right)(x) \\
& +T\left(\left(b_{1}-\lambda_{1}\right) f_{1}^{\infty},\left(b_{2}-\lambda_{2}\right) f_{2}^{\infty}\right)(x) \\
= & I V_{1}+I V_{2}+I V_{3}+I V_{4} .
\end{aligned}
$$

Because each term of $I V_{j}$ is completely analogous to $I I_{j}, j=$ $1,2,3,4$ with a bit difference, so we get the following estimate without details:

$$
\left(\frac{1}{v_{\vec{\omega}}(Q)^{k}} \int_{Q}|I V|^{p} v_{\vec{\omega}}(x) d x\right)^{1 / p} \leq C \prod_{j=1}^{2}\left\|b_{j}\right\|_{\mathrm{BMO}}\left\|f_{j}\right\|_{L^{p_{j}, k}\left(\omega_{j}\right)} .
$$

To this, we end the proof of Theorem 9.

\section{Conflict of Interests}

The authors declare that they have no conflict of interests.

\section{Acknowledgment}

This research was supported by NSF of China (no. 11161044, no. 11261055) and by XJUBSCX-2012004. 


\section{References}

[1] L. Grafakos and R. H. Torres, "Multilinear Calderón-Zygmund theory," Advances in Mathematics, vol. 165, no. 1, pp. 124-164, 2002.

[2] C. Pérez, G. Pradolini, R. H. Torres, and R. T. Trujillo-Gonza'lez, "End-point estimates for iterated commutators for multilinear singular integrals," Bulletin London Mathematical Society, 2013.

[3] C. Pérez and R. H. Torres, "Sharp maximal function estimates for multilinear singular integrals," Contemporary Mathematics, vol. 320, pp. 323-331, 2003.

[4] X. T. Duong, L. Grafakos, and L. Yan, "Multilinear operators with non-smooth kernels and commutators of singular integrals," Transactions of the American Mathematical Society, vol. 362, no. 4, pp. 2089-2113, 2010.

[5] X. T. Duong, R. Gong, L. Grafakos, J. Li, and L. Yan, "Maximal operator for multilinear singular integrals with non-smooth kernels," Indiana University Mathematics Journal, vol. 58, no. 6, pp. 2517-2541, 2009.

[6] B. T. Anh and X. T. Duong, "On commutators of vector BMO functions and multilinear singular integrals with non-smooth kernels," Journal of Mathematical Analysis and Applications, vol. 371, no. 1, pp. 80-94, 2010.

[7] A. K. Lerner, S. Ombrosi, C. Pérez, R. H. Torres, and R. TrujilloGonzález, "New maximal functions and multiple weights for the multilinear Calderón-Zygmund theory," Advances in Mathematics, vol. 220, no. 4, pp. 1222-1264, 2009.

[8] L. Grafakos, L. G. Liu, and D. C. Yang, "Multiple weighted norm inequalities for maximal multilinear multilinear singular integrals with non-smooth kernels," Proceedings of the Royal Society of Edinburgh A, vol. 141, pp. 755-777, 2011.

[9] S. Chen and H. Wu, "Multiple weighted estimates for commutators of multilinear singular integrals with non-smooth kernels," Journal of Mathematical Analysis and Applications, vol. 396, no. 2, pp. 888-903, 2012.

[10] Y. Komori and S. Shirai, "Weighted Morrey spaces and a singular integral operator," Mathematische Nachrichten, vol. 282, no. 2, pp. 219-231, 2009.

[11] Q. Xue and J. Yan, "Multilinear version of reversed Hölder inequality and its applications to multilinear CalderónZygmund operators," Journal of the Mathematical Society of Japan, vol. 64, no. 4, pp. 1053-1069, 2012.

[12] J. Garcia-Cuervaand and J. L. Rubio de Francia, Weighted Norm Inequalities and Related Topics, vol. 116 of North-Holland Mathematics Studies, North-Holland, Amsterdam, The Netherlands, 1985. 


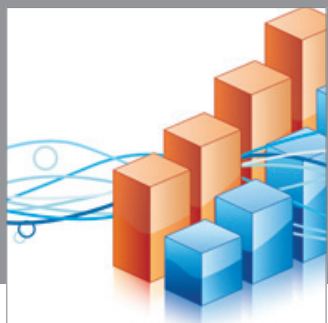

Advances in

Operations Research

mansans

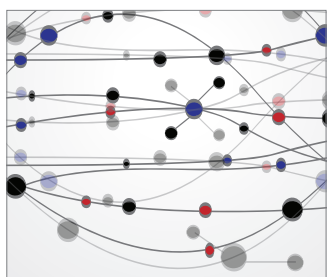

The Scientific World Journal
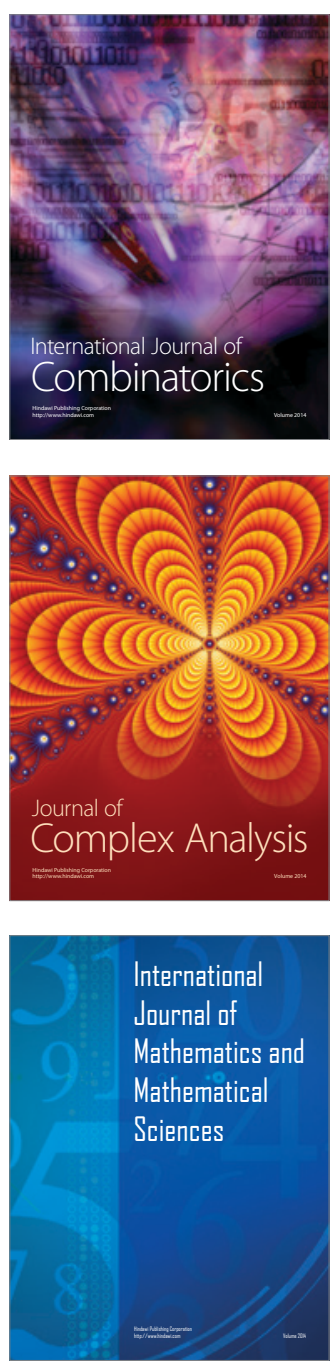
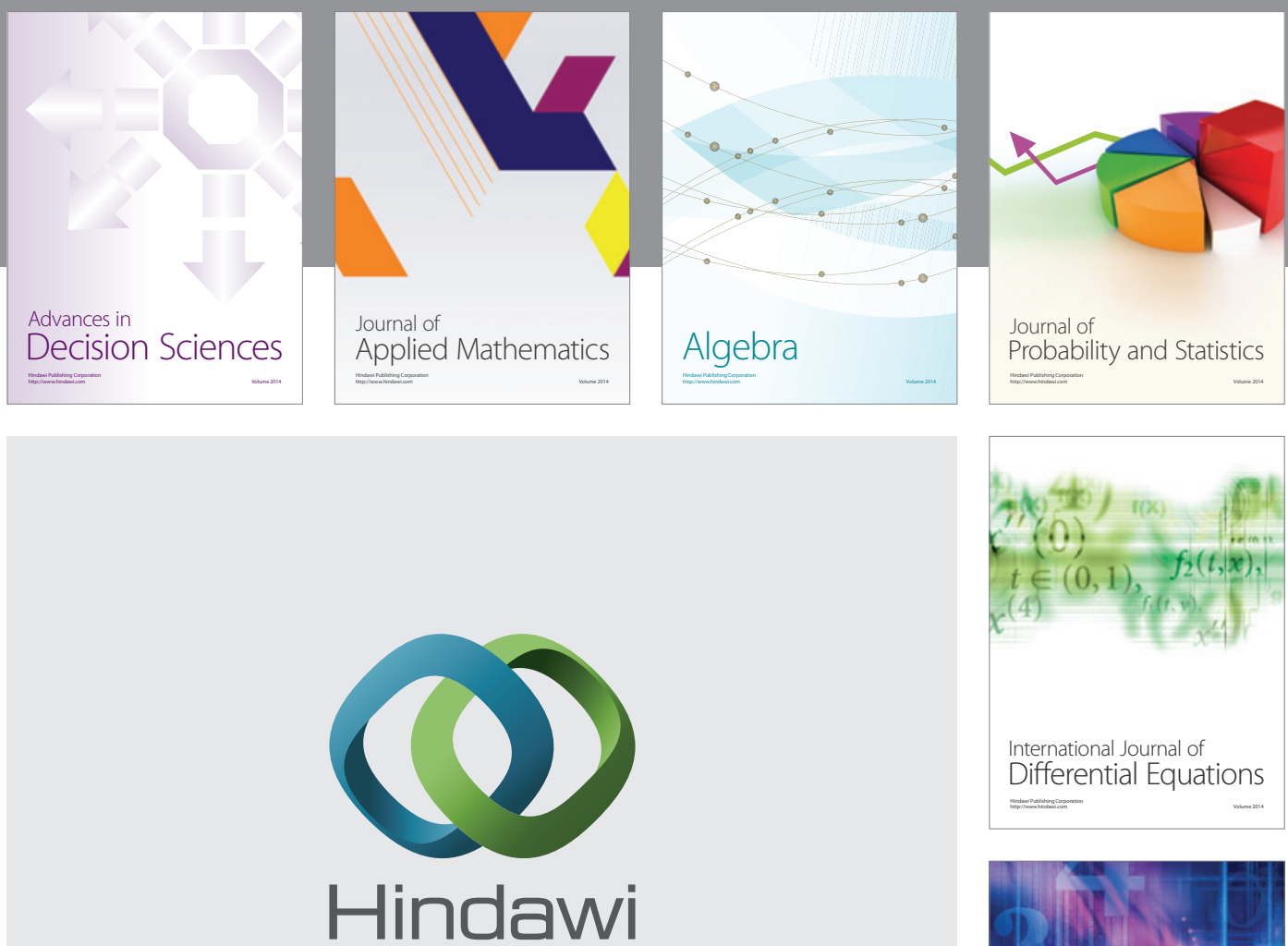

Submit your manuscripts at http://www.hindawi.com
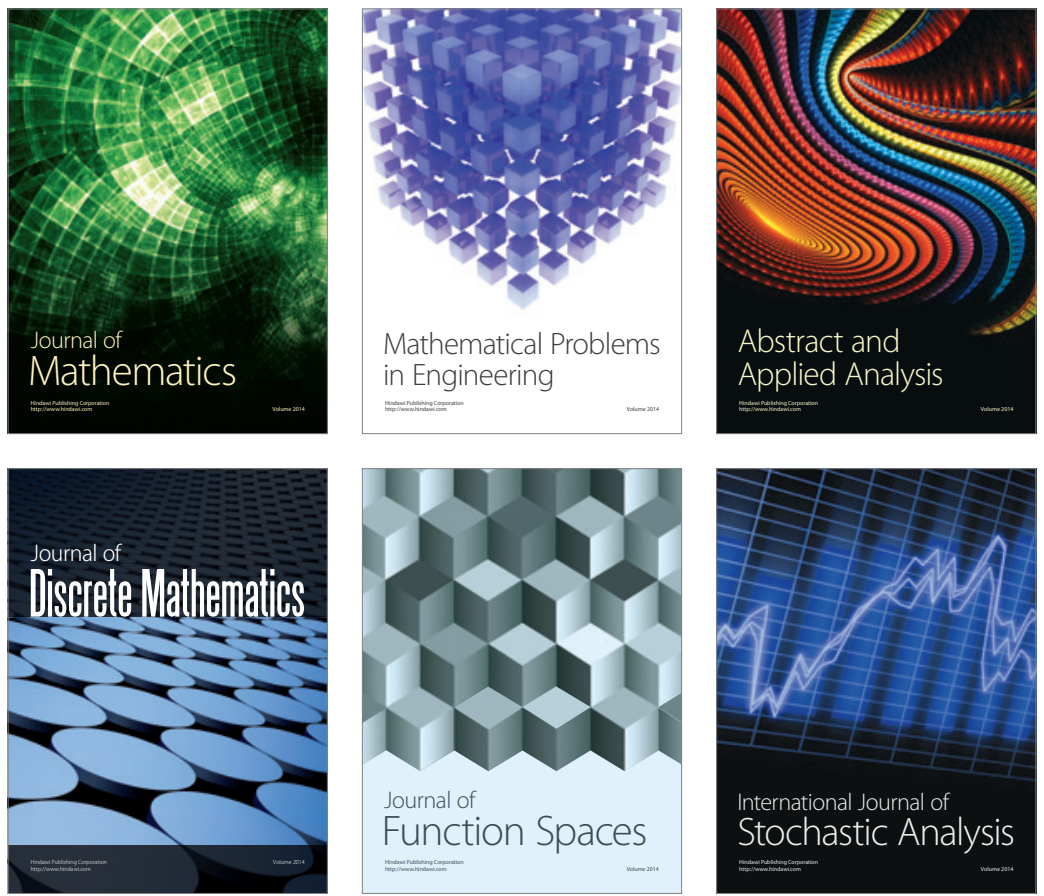

Journal of

Function Spaces

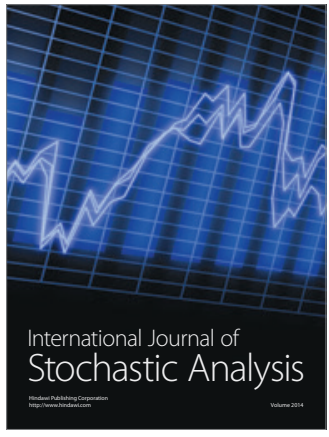

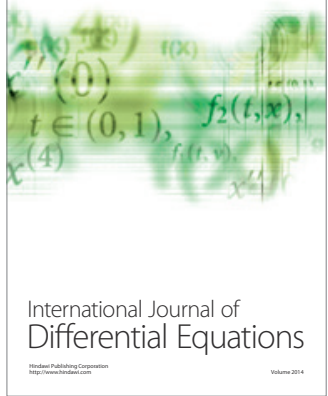
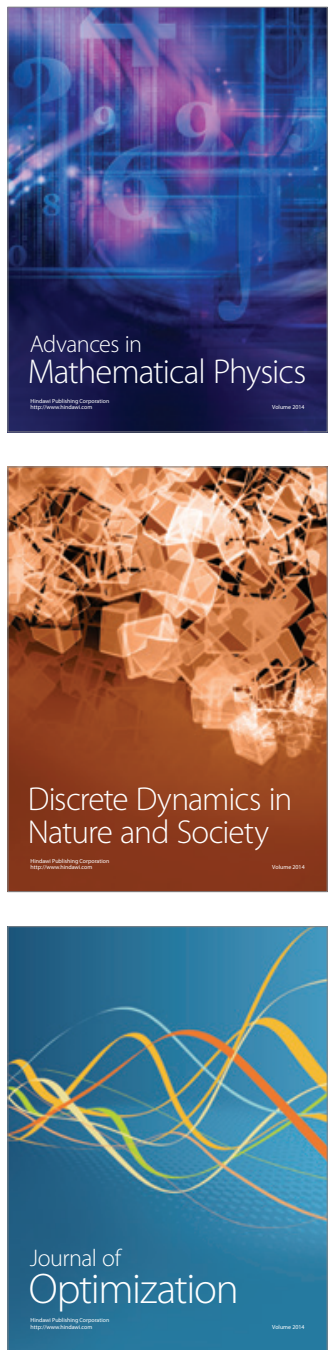